\title{
FROM THE SLIT-ISLAND METHOD TO THE ISING MODEL: ANALYSIS OF IRREGULAR GRAYSCALE OBJECTS
}

\author{
PRZEMYSŁAW MAZUREK*, DOROTA OSZUTOWSKA-MAZUREK *,** \\ * Department of Signal Processing and Multimedia Engineering \\ West-Pomeranian University of Technology, ul. 26 Kwietnia 10, 71-126 Szczecin, Poland \\ e-mail:przemyslaw.mazurek@zut.edu.pl \\ ** Department of Pathomorphology \\ Gryfice Hospital Medicam, Niechorska 27, 72-300 Gryfice, Poland \\ e-mail:adorotta@op.pl
}

\begin{abstract}
The Slit Island Method (SIM) is a technique for the estimation of the fractal dimension of an object by determining the areaperimeter relations for successive slits. The SIM could be applied for image analysis of irregular grayscale objects and their classification using the fractal dimension. It is known that this technique is not functional in some cases. It is emphasized in this paper that for specific objects a negative or an infinite fractal dimension could be obtained. The transformation of the input image data from unipolar to bipolar gives a possibility of reformulated image analysis using the Ising model context. The polynomial approximation of the obtained area-perimeter curve allows object classification. The proposed technique is applied to the images of cervical cell nuclei (Papanicolaou smears) for the preclassification of the correct and atypical cells.
\end{abstract}

Keywords: slit island method, area-perimeter method, Ising model, image analysis, cervical cancer.

\section{Introduction}

There are numerous techniques for the description of object shape and texture. The typical parameters are the area, perimeter, Fourier descriptors and the Ferret diameter. Loncaric (1998), Zieliński and Strzelecki (2002), Mingqiang et al. (2008), Smereka and Dulęba (2008), Costa and Cesar Jr. (2009) as well as Śmietański et al. (2010) showed more than a hundred object descriptors, related to the shape. New descriptors are still being developed. The description of the texture is more complex: statistical parameters, spatial relations and multiscale techniques are applied (Engler and Randle, 2010).

The estimation of the Fractal Dimension (FD) is very important for pattern recognition theory and its applications. The FD creates a bridge between local and global object characteristics. Original works related to the FD are focused on the estimation of the single value FD (Mandelbrot, 1983; Peitgen et al., 1991; 1992). The single FD value is not feasible for real applications, because FD changes are observed depending on the scale. This is typical for many natural objects (Kaye, 1994; Barnsley et al., 1988). The estimation of the FD is very important for large scale and microworld object descriptions (Seuront, 2010; Kaye, 1994; Mandelbrot, 1983). The FD allows the estimation of the object's parameters and further computer synthesis of similar objects that is used in computer graphics, for example.

The concept of the FD is based on non-integer dimensions (Mandelbrot, 1983; Peitgen et al., 1991). There are many definitions of the dimension, and some of them allow fractional values of the dimension. The fractional dimension is the effect of higher space filling abilities of an object. A single curve (1D object) fills a $2 \mathrm{D}$ area, so it behaves as an object that has a non-integer dimension with a value between 1 and 2 . A similar concept is related to $2 \mathrm{D}$ surfaces which fill the $3 \mathrm{D}$ space partially.

The most well-known FD estimators are the coastline stick, area (e.g., box-counting), or volume (Mandelbrot, 1983; Peitgen et al., 1991). Estimation of the FD is based on the analysis of the space filling by the line, square or box, depending on the scale. The scale is defined by the size of the filling element. Changes in FD values could be obtained depending on the scale. The graphical representation in the double logarithmic plot is known 
as the Richardson plot (Mandelbrot, 1983; Peitgen et al., 1991; Steven, 1993). The slope of the regression line from the Richardson plot is used for the computation of the FD, but some additional operations are desired for the specific FD estimator. Many natural objects change the FD depending on the scale, so local regression lines should be used, and at least two values are obtained (Kaye, 1994; Seuront, 2010; Harte, 2001).

FD estimation is an efficient technique for object analysis and classification. Many types of objects are characterized by a single FD value. Complex objects require multiple FDs (Kaye, 1994; Seuront, 2010; Harte, 2001), which are scale dependent. Some non-fractal parameters are scale independent, but scale dependent parameters are also available. A combination of the FD and non-fractal descriptors should also be considered an important set of estimators.

1.1. Paper organisation. Various fractal dimension estimators for grayscale images are considered briefly and an example of the cell nucleus optical density is shown in Section 2

The application of the known Slit-Island Method (SIM) for grayscale image analysis is considered in Section 3 The main limitations of this method are emphasized, like influence on the selection of the boundary analysis approach and inadequate results of FDs estimation, where infinite or negative FD could be obtained.

The proposed approach for area-perimeter analysis is considered in Section 4 instead of the rejection of the SIM. The connection between the SIM and the 2D Ising model is introduced. The magnetization curve for the grayscale image could be obtained and the pseudo-Curie point could be determined.

The shape of the magnetization curve for grayscale image could be applied for the object's parameters estimation. The obtained parameters could be applied for further analysis and classification of objects from the database.

The proposed area-perimeter analysis in the context of the magnetization curve is applied as an example for the analysis of cytological images of cell nuclei in Section 5. The magnetization curve is approximated using polynomials. The relations of polynomial coefficients are applied for classification purposes. Sensitivity analysis using eroded images is also considered. It is especially important, because cell nuclei segmentation is difficult due to the inherent low quality of the images.

1.2. Related works and contribution of the paper. Fractal analysis of grayscale images is possible using many algorithms: the TPM (Triangular Prism Method) (Clarke, 1986) and derivatives (Sun, 2006), the variogram (Atkinson, 2002; Kaye, 1994), the isarithm (Goodchild, 1980), the variation estimator (Parker, 1997), as well as the probability estimator (Voss, 1988).

The analysis of grayscale images is possible using the SIM (Mandelbrot et al., 1984; Mandelbrot, 1983), as an unordered set of binary images (layers). The perimeter and area are estimated for every layer. This technique is applied by many researchers. Some of them consider limitations or even rejection of this technique.

In this paper, significant limitations of the SIM are shown through illustrative examples. The rejection of the SIM is not emphasized intentionally, because this technique has a great potential of being valuable, if a proper modification is applied. The modification of the SIM allows the analysis of grayscale images. The proposed layer number that corresponds to the threshold level gives the area-perimeter relation. This relation could be modified by the replacement of the unipolar binary image representation, used in the original SIM, by the bipolar binary image representation, which is proposed in this paper. This modification establishes a link between fractal analysis using the original SIM and another important image analysis technique-the Ising model. The area-perimeter relation in the context of the Ising model allows the introduction of the pseudo-Curie point, which could be used for classification purposes.

The proposed approach is applied for the preclassification of biological objects. The test is related to the possible application to cervical cancer diagnosis. The cell nuclei of Papanicolaou strains are considered instead of more complex analysis using, e.g., the NC (Nuclear/Cytoplasmic) ratio, where the segmentations of the cell nuclei and cytoplasm are necessary. Cytoplasm segmentation is a very difficult task due to cytoplasm overlapping in cell clusters.

The cell nucleus image is obtained using transmissive microscopy, and the optical density of a $3 \mathrm{D}$ object is acquired as a $2 \mathrm{D}$ image. The analysis of the 3D shape of cell nuclei using, e.g., the 3D from focus technique is beyond the paper scope. The classification of cell nuclei using a single image is faster and sufficient. The database contains many atypical cell nuclei that are rare cases obtained from a 2-year screening period in a medium scale laboratory. Cytodiagnosis screening programs reduced the occurrence of cancer, but the detection of atypical cells is very sophisticated (there are many types of atypia). Cervical cancer is not a health problem separately, but the source of many social and psychological problems for patients if not detected in an early stage. 


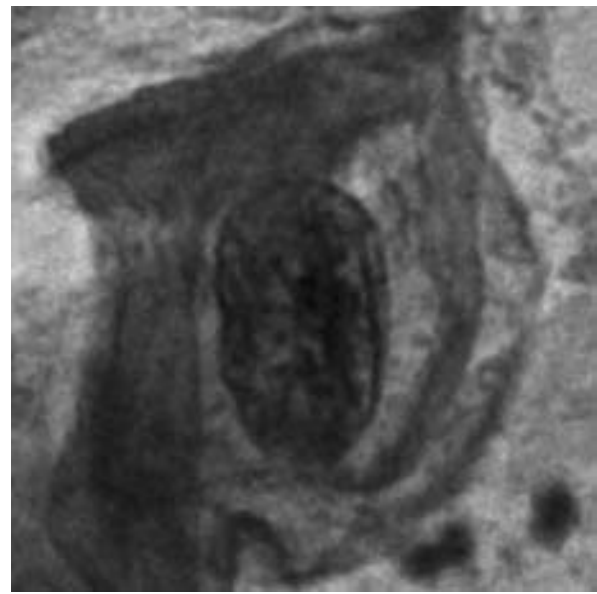

Fig. 1. Microscope image of a cervical uteri cell with an atypical cell nucleus.

\section{Fractal dimension estimators for grayscale images}

There are many FD estimators for binary images, e.g., the ones based boxcounting. Multivalued (grayscale) images need dedicated FD estimators because the object representation could be three-dimensional. Some examples were investigated: the TPM (Clarke, 1986) and derivatives (Sun, 2006), the variogram (Atkinson, 2002; Kaye, 1994), the isarithm (Goodchild, 1980), the variation estimator (Parker, 1997), the probability estimator (Voss, 1988).

Overestimation and underestimation of the FD for synthetically generated fractals is a well-known fact (Wen and Sinding-Larsen, 1997; Zhou and Lam, 2005). Such estimation errors are not important for pattern recognition applications, if the monotonicity of the estimation is preserved for the particular method.

The estimation of the FD is straightforward for square images. Some constraints should be fulfilled for specific methods, related to the width of the square side. A typical requirement is the side length of the square, $\left(2^{p}+\right.$ 1 ) or $2^{p}$, where $p$ corresponds to the scale. Non-square objects cannot be properly processed directly. Areas that do not belong to the object influence the FD. Image area reduction considered for FD estimation introduces errors, unfortunately. Better fitting to the object area is possible by the applications of tiled processing (e.g., the tiled TPM algorithm (Oszutowska and Purczyński, 2012)), where smaller portions of the object are used for FD estimation instead of single square with a largest available area.

A cytological image of the cell, with the object of, interest, i.e., a cell nucleus, is shown in Fig. 1 . The 3D representation of the optical density of the cell nucleus is shown in Fig. 2 for the blue channel. Such a surface is the DEM (Digital Elevation Map), processed by, e.g., the variogram (Oszutowska-Mazurek et al., 2013)

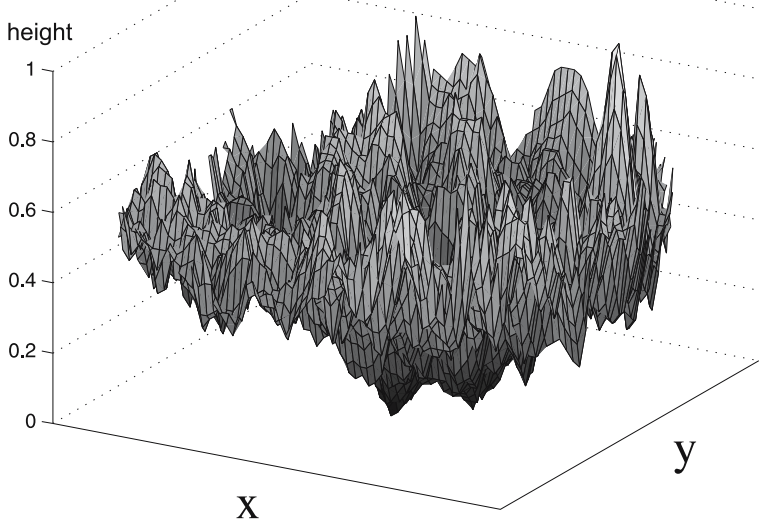

Fig. 2. Three-dimensional visualization of the optical density of the cell nucleus (background pixels are omitted) from Fig. 1.

and TPM methods (Oszutowska and Purczyński, 2012; Oszutowska-Mazurek et al., 2012).

\section{Slit-island method}

3.1. Introduction. The slit-island method, known also as the slit-island technique or the area-perimeter method (Mandelbrot et al., 1984; Mandelbrot, 1983), could be applied for grayscale images. The application to the grayscale image $I$ is possible by the conversion to multiple layers (binary valued) using thresholding:

$$
X^{T}(x, y)= \begin{cases}1 & \text { if } \quad I(x, y)<T \\ 0 & \text { if } \quad I(x, y) \geq T\end{cases}
$$

The threshold value $T$ defines a particular layer. The SIM does not specify requirements related to the image $I$ or the threshold level $T$, which is an open problem.

The binary image $X^{T}$, related to the particular threshold, is processed and all islands, marked by $i$, are analyzed. The relation of the perimeter $P_{i}$ to the area $A_{i}$ gives a constant value ratio $C$, and it is defined by the following formula:

$$
C^{T}(r)=\frac{\left(\sum_{i} P_{i}^{T}(r)\right)^{1 / D_{f}^{\prime}}}{\left(\sum_{i} A_{i}^{T}(r)\right)^{1 / 2}},
$$

where $D_{f}$ is the fractal dimension,

$$
D_{f}=D_{f}^{\prime}-1 \text {. }
$$

Such a dimension is independent of the length of yardstick $r$ used for the measurement. A fixed yardstick is assumed, which is convenient for digital images. In this case, $r$ is equal to the pixel width/height size $(r=1)$. For a monofractal, $D_{f}$ is not a function of layer $(T)$, but in real 
cases it is a function of the layer number $\left(D_{f}^{T}\right)$, which is discussed later.

The following formula uses the summation over all islands for the perimeter and area, so the computation of the area is based on the counting of pixels of specific values, e.g., the $1 \mathrm{~s}$ :

$$
\sum_{i} A_{i}^{T}(r)=\sum_{x, y} X^{T}(x, y)
$$

where $x$ and $y$ denote the 2D coordinates on the image $X$. It is assumed that the value of 1 belongs to the pixel of the island. The computation of the perimeter is possible using the following formula:

$$
\begin{aligned}
\sum_{i} P_{i}^{T}(r)= & \frac{1}{2} \sum_{x, y}\left(X^{T}(x, y) \wedge \overline{X^{T}(x, y-1)}\right. \\
& +X^{T}(x, y) \wedge \overline{X^{T}(x, y+1)} \\
& +X^{T}(x, y) \wedge \overline{X^{T}(x-1, y)} \\
& \left.+X^{T}(x, y) \wedge \overline{X^{T}(x+1, y)}\right) .
\end{aligned}
$$

This formula allows the calculation of cliques between two adjacent pixels. The edge exists if neighborhood pixels values are opposite. The summation of edges allows the estimation of the cumulative perimeter. A scaling coefficient $(1 / 2)$ is necessary, because a single clique is detected and counted twice.

The SIM is applied to the multiple layers $T$ of the object, so successive sections of the 3D object are obtained (Kaye, 1994; Mandelbrot et al., 1984; Mandelbrot, 1983). The measurement of the cumulative perimeter and area is plotted on a double logarithmic plot. The slope of the regression line allows the estimation of the FD. Example layers for the cell nucleus DEM are shown in Fig. 3 but precise estimation of the FD should be based on tens or hundreds of layers.

The SIM requires one very important assumption that is not emphasized in literature. No islands can be located on the boundary of the image. The relation between the area and perimeter for an example object is shown in Fig. 4. It is clear that the estimation of the FD for the example object is very problematic. The curve is not a straight line. The regression line cannot be properly established. There are some parts of the curve where the regression line should be ascending, descending or even vertical. Those problems are considered in the next subsections.

3.2. Boundary area. The SIM is very sensitive - the boundary area of the image (the first and last row and column of the image) influences on the results. A partially available island limited the area and perimeter, so the result is biased. In many applications, object images are obtained by the successive cutting or polishing of the

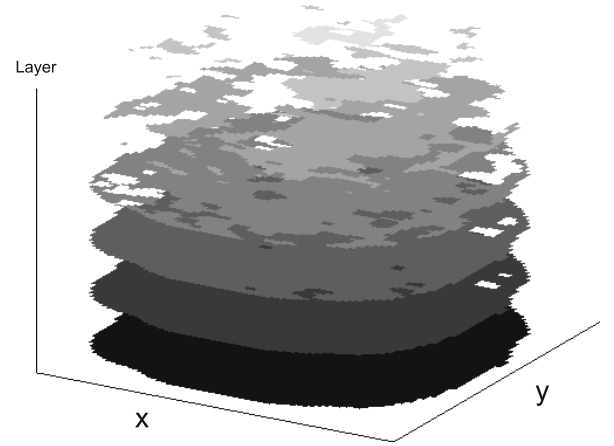

Fig. 3. Layers of the cell nucleus representation from Fig. 2

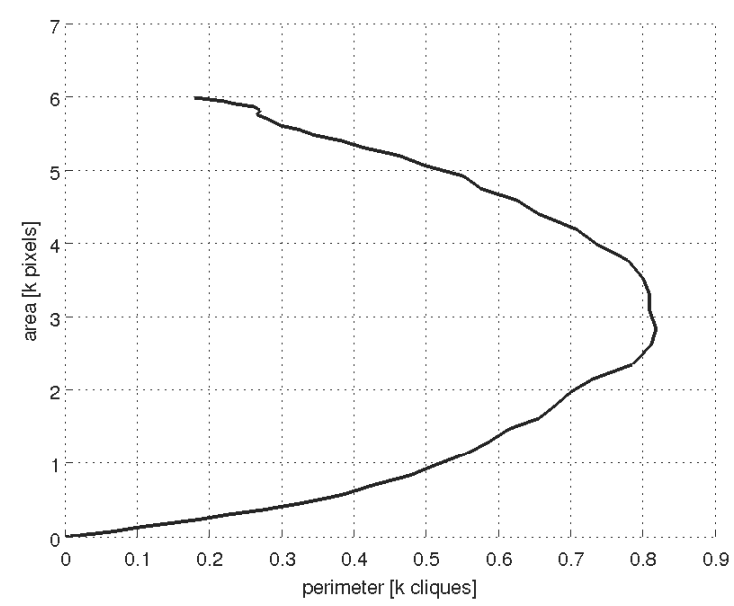

Fig. 4. Area-perimeter relation for the cell nucleus from Fig. 1

sample, so the rejection of boundary islands cannot be fulfilled due to physical properties of the object. The rejection of such objects is possible for a single layer, but rejection for multiple layers could be very sophisticated because connection between islands on layers could be considered. Some classes of objects create connections between islands that are a merger for the lower layer to the single area. The rejection of such island is the rejection of the possibility of estimation.

The second influence is related to the perimeter computation technique. Assuming a non-rectangular sample, there are three areas: islands, lakes and the background. The background is related to object image storage inside the rectangular area.

A simple assignment of the pixel (1s to the islands, 0 s to the lakes and background) is very attractive and straightforward but influences the results. The clique between the island pixel and background pixel increases the perimeter. The perimeter is calculated using the formula (5). Results are shown, for example, in Fig. 4

An alternative assignment of a pixel (1s to the island, 
Os to the lakes) is more adequate to the relation between pixels of the object. Background pixels are assigned to the third (forbidden) value $B$ (Fig. 5). The clique between the island and background does not increase the perimeter. The following formula is applied for the calculation of the perimeter:

$$
\begin{aligned}
& \sum_{i} P_{i}^{T}(r)=\frac{1}{2} \sum_{x, y}\left(C_{1}+C_{2}+C_{3}+C_{4}\right), \\
& C_{1}= X^{T}(x, y) \wedge \overline{X^{T}(x, y-1)} \wedge \cdots \\
& \cdots X^{T}(x, y) \neq B \wedge X^{T}(x, y-1) \neq B, \\
& C_{2}= X^{T}(x, y) \wedge \overline{X^{T}(x, y+1)} \wedge \cdots \\
& \cdots X^{T}(x, y) \neq B \wedge X^{T}(x, y+1) \neq B, \\
& C_{3}= X^{T}(x, y) \wedge \overline{X^{T}(x-1, y)} \wedge \cdots \\
& \cdots X^{T}(x, y) \neq B \wedge X^{T}(x-1, y) \neq B, \\
& C_{4}= X^{T}(x, y) \wedge \overline{X^{T}(x+1, y)} \wedge \cdots \\
& \cdots X^{T}(x, y) \neq B \wedge X^{T}(x+1, y) \neq B .
\end{aligned}
$$

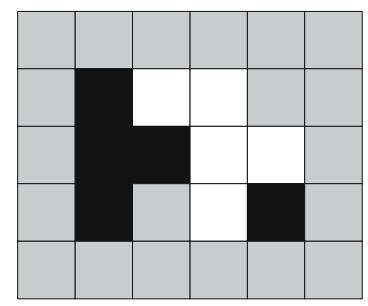

\begin{tabular}{|l|l|l|l|l|l|}
\hline 0 & 0 & 0 & 0 & 0 & 0 \\
\hline 0 & 1 & 0 & 0 & 0 & 0 \\
\hline 0 & 1 & 1 & 0 & 0 & 0 \\
\hline 0 & 1 & 0 & 0 & 1 & 0 \\
\hline 0 & 0 & 0 & 0 & 0 & 0 \\
\hline
\end{tabular}

\begin{tabular}{|c|c|c|c|c|c|}
\hline B & B & B & B & B & B \\
\hline B & 1 & 0 & 0 & B & B \\
\hline B & 1 & 1 & 0 & 0 & B \\
\hline B & 1 & B & 0 & 1 & B \\
\hline B & B & B & B & B & B \\
\hline
\end{tabular}

Fig. 5. Example image (top), two level $\{0,1\}$ (bottom left), three level $\{0,1, B\}$ (bottom right) representations of an irregular object (0: lake, 1: island, B: background pixel. Perimeter cliques are emphasized by the dashed line).

The three level variant gives different results (Fig. 6) in comparison with the two-level variant (Fig. 4). Artificial boundaries between islands and the boundary are not processed, so some symmetry of the area-perimeter curve is achieved (Fig. 6).

3.3. Infinite FD. The estimation of the FD should be possible for any image. There are some objects that show problems of the SIM. Assuming the starting image (layer) $X_{0}$ the satisfying

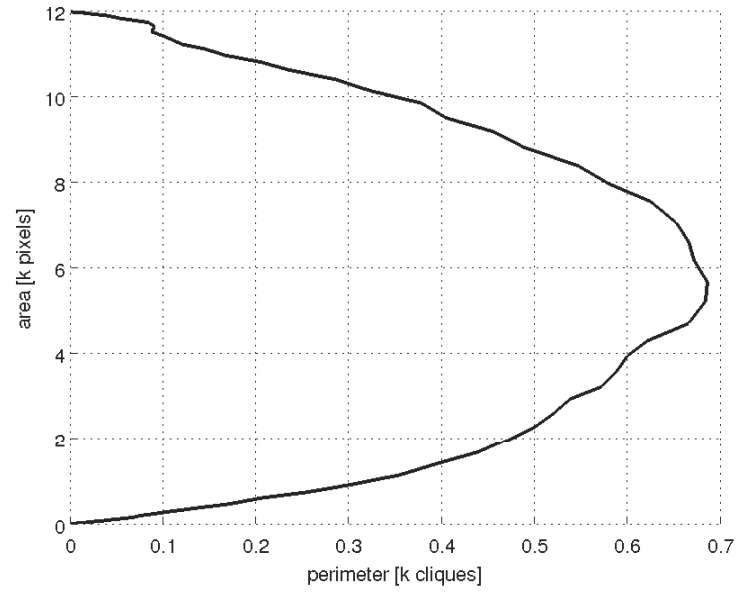

Fig. 6. Area-perimeter relation for three level representation for the cell nucleus from Fig. 1.

$$
X_{0}(x, y)= \begin{cases}1 & \text { if } x=y \wedge x, y>1 \wedge x, y<N \\ 0 & \text { otherwise }\end{cases}
$$

where the image has $N \times N$ resolution and $x, y \in[1, N]$ are coordinates, a diagonal line is obtained. The boundary of this image is filled with zeros. The perimeter of this object is equal to $4(N-2)$ and the area is $(N-2)$. The next images (layers of the grayscale image) are created using a morphological operator or cellular automata. Switching the pixel value to 1 is possible if there are two adjacent pixels with value 1 , for the 4-way neighborhood. The following morphological operation could be applied:

$$
\begin{aligned}
& X_{n+1}=X_{n} \oplus O P_{i}, \\
& O P_{1}=\left[\begin{array}{ll}
1 & x \\
\underline{0} & 1
\end{array}\right], \\
& O P_{2}=\left[\begin{array}{ll}
1 & \underline{0} \\
x & 1
\end{array}\right], \\
& O P_{3}=\left[\begin{array}{ll}
\underline{0} & 1 \\
1 & x
\end{array}\right], \\
& O P_{4}=\left[\begin{array}{ll}
x & 1 \\
1 & \underline{0}
\end{array}\right] .
\end{aligned}
$$

It is a kind of dilation. Only two operators are useful, depending on the axes of image orientation. Two others give no line expansion. One correct operator gives the expansion toward the specific direction. The second correct operator gives the expansion towards the opposite direction

Multiple iterations of such dilation are necessarily related to a single pixel, using any update strategy 
(deterministic or stochastic). After some number of operations, a box filled with $1 \mathrm{~s}$ is obtained. Every successful operation of switching the pixel value to 1 , according to the morphological formula, increases the object area. The final box has the width of one side equal to $(N-2)^{2}$ and the there are no perimeter changes at all. The perimeter is always equal to $4 \cdot(N-2)$. A single operation on a pixel is depicted in Fig. 77 A few example results are shown in Fig. 8,
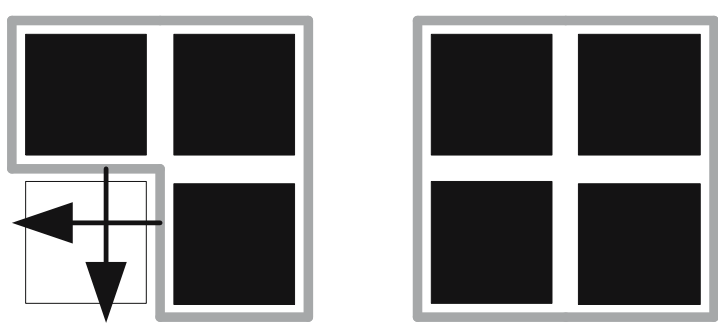

Fig. 7. Example of dilation without perimeter changes for a specific pixel $\left(P_{i}=8, A_{i}=3\right.$ and $\left.P_{i+1}=8, A_{i+1}=4\right)$.
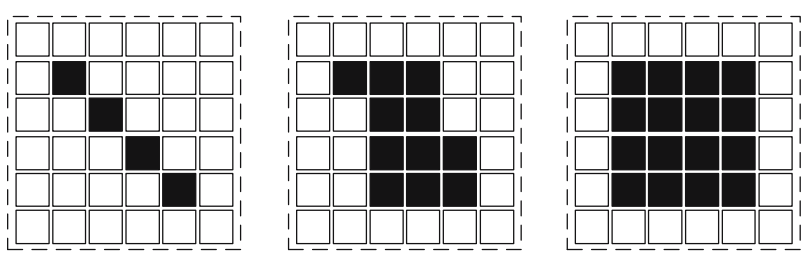

Fig. 8. Initial $\left(P_{0}=16, A_{0}=4\right)$, intermediate $\left(P_{i}=16, A_{i}=\right.$ $11)$ and final $\left(P_{N}=16, A_{N}=16\right)$ dilation of the diagonal line.

The estimation of the FD using a regression line analysis is possible, and the value of the FD is infinity because the regression line is vertical. This example shows that a simple object with specific layers cannot be processed using the SIM. Real images may preserve some behaviors of this example and overestimate the FD.

3.4. Negative FD for an island with lakes. Islands with lakes are very important. An island with a lake could be a source of a negative FD. It is possible to obtain a reduction in the perimeter and expansion of the area if the starting image is a rectangle frame. The choice of lakes within islands is questioned, e.g., by Huang et al. (1990).

The rectangle frame, with $1 \mathrm{~s}$ at edges and zero filled image boundaries, will evolve to the filled rectangle. The initialization pattern is

$$
X_{0}(x, y)= \begin{cases}0 & \text { if } \quad x, y>2 \wedge x, y<N-1, \\ 0 & \text { if } x=1 \vee x=N \vee y=1 \vee y=N \\ 1 & \text { otherwise }\end{cases}
$$

The first zero case is related to the inner part of the rectangle and the second zero case is related to the boundary of the image.
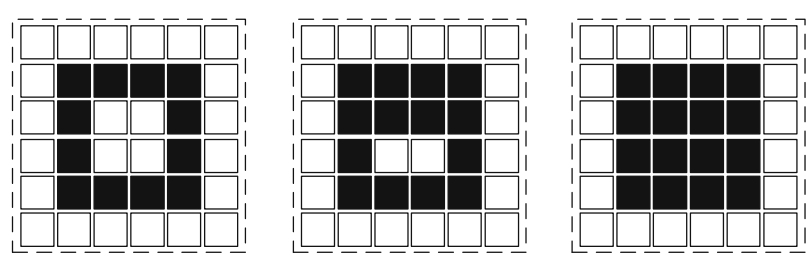

Fig. 9. Initial ( $\left.P_{0}=24, A_{0}=12\right)$, intermediate $\left(P_{i}=\right.$ $\left.22, A_{i}=14\right)$ and final $\left(P_{N}=16, A_{N}=16\right)$ dilation of the rectangle.

Different types of islands, including an island with lakes, may give noisy results, so the SIM cannot be applied without additional assumptions. The closing of lakes within islands is typical for binary images filled with many 1s. Such an effect is typical for grayscale images with multiple local maximums. The size of lakes is increased, moving toward the bottom layers, for a blurred image. Merging lakes is the source of the creation of large islands with lakes inside them. Such lakes are closed in the next bottom layers, so negative FD measurement results are obtained.

Self-affine patterns create multiple FDs (Lu, 1995), so only self similar islands should be processed. This is a limitation of the SIM, which reduces application area to specific objects only.

We would like to show the connectivity of the SIM and the Ising model, which allows application of area-perimeter analysis for more general classes of objects, instead of the criticism of SIM only. The extension of the SIM approach, where the FD is replaced by a different estimator, is possible and desired, instead of the SIM rejection only. The connection between them is very interesting, because similar works of other researchers in this area are not known.

\section{Reformulation of the SIM in the Ising model context}

4.1. Spin based model of image. The SIM is based on binary image analysis, where one of two unipolar values is assigned to pixels:

$$
X_{\mathrm{SIM}}(x, y) \in\{0,1\} .
$$




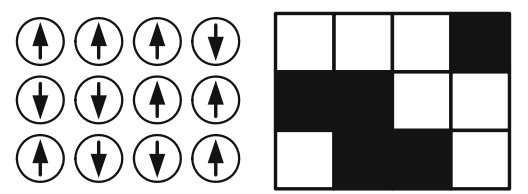

Fig. 10. Example of a finite size Ising model and the relation to a binary image.

The replacement of such values by complementary (bipolar) values gives the opportunity of area-perimeter analysis reformulation in a different context:

$$
X(x, y) \in\{-1,+1\}
$$

The lattice $X$ corresponds to a $2 \mathrm{D}$ crystal network. The pixel values are spin values (Skomski, 2008), which is depicted in Fig. 10 Statistical mechanics allows the description of such a structure using many models (Skomski, 2008; Styer, 2007), and the most important is the 2D Ising model.

The Ising model is important for the description of ferromagnetic properties of the lattice and supports transitions of the II-type of critical phenomena (Binney et al., 1992).

4.2. Magnetization. Overall magnetization is related to the internal magnetic field, defined by the orientation of the spins. The magnetization is the sum of the spins

$$
M=\sum_{x, y} X(x, y)
$$

which is equivalent to $\sum_{i} A_{i}(r)$. The difference is the offset coefficient $K$

$$
2 \sum_{i} A_{i}(r)-K=\sum_{x, y} X(x, y),
$$

where $K$ is the number of spins (pixels). The state where all pixels are zero (unipolar) corresponds to the case where all spins are directed down -1 (bipolar). The offset coefficient $K$ is responsible for value shift. Such a formulation for bipolar representation ensures the symmetry for two opposite cases of magnetization:

$$
M_{\max }=-M_{\min }=K \text {. }
$$

4.3. Hamiltonian. The Hamiltonian (Skomski, 2008) is the total energy of a system. It governs the dynamics of the system. The Hamiltonian for the Ising model is very simple:

$$
H=-\frac{1}{2} \sum_{i, j} w_{i, j} X_{i} X_{j}-h^{\mathrm{EXT}} \sum_{i} X_{i} .
$$

Two parts are considered in the formula and related to the interaction between lattice sites and between the external field. Every lattice site (pixel) has four neighborhoods.

The external field is not available for the case considered, so the assumption $h^{\mathrm{EXT}}=0$ is allowed. It is one of the most well investigated random cluster models that support 'ferromagnetic' properties. The following formula corresponds to the number of cliques:

$$
H=-\frac{1}{2} \sum_{i, j} w_{i, j} X_{i} X_{j},
$$

where the scaling coefficient $1 / 2$ is necessary due to the removal of the double counting of cliques. The weight $w_{i, j}$ is responsible for the set-up of, e.g., 4-way connectivity. The relation between the Hamiltonian and perimeter is finally

$$
-H=P \text {. }
$$

4.4. Glauber dynamics. Thermal fluctuations in the Ising model are specified by the Glauber dynamics and simulated using, e.g., a Gibbs sampler or a heat-bath algorithm (Glauber, 1963). The stochastic rule of changes is defined using the following formula:

$$
X(x, y)=\left\{\begin{array}{lll}
+1 & \text { with probability } & g(h), \\
-1 & \text { with probability } & 1-g(h) .
\end{array}\right.
$$

The function $g(h)$ is temperature dependent. According to Glauber (1963), the following sigmoid function should be used:

$$
g(h)=\frac{1}{\exp (-2 \beta h)},
$$

where $\beta$ is the reciprocal of the absolute temperature

$$
\beta=\frac{1}{k_{B} T} .
$$

The Boltzmann constant is marked as $k_{B}$, but it is a physical constant. This formula for image processing applications is not necessary, so $\beta$ is used only or $k_{B}=1$ is assumed for simplification.

4.5. Curie temperature. The Curie temperature $\left(T_{C}\right)$ is a temperature $(T)$ when the internal magnetization is lost, which means that

$$
M\left(T_{C}\right)=0
$$

This is a singularity point and such behavior is specific for ferromagnetic materials and occurs in phase II-type transitions only. The curve $M=f(T)$ is symmetrical around $T$-axis, additionally. There are two branches of this function: upper and lower (Fig. 11). A 
single branch is depicted in most papers and books, but both are very important for image analysis. The upper branch is related to the image mostly filled with, e.g., white pixels, and the lower branch is related to the image mostly filled with black pixels.

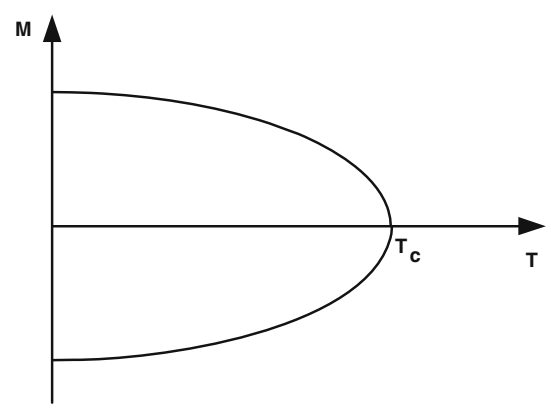

Fig. 11. Magnetization curve (both branches are shown).

The Glauber process defines the time evolution of spin latices (images). A sequence of such images is driven stochastically (26), depending on the assumed temperature (27), (28). Fluctuations in the perimeter and area of these binary images are achieved. Temperature changes influence creation specific structures, e.g., magnetic domains.

Changes in the temperature allow movements from $-M_{\min }$ to the Curie point $M_{0}$ and, further, to $+M_{\max }$. This trajectory correspond to changes from $-A_{\min }$ to $A=0$ and further to $+A_{\max }$, depending on the perimeter (energy of cliques) for grayscale image layers. The relation between magnetization and the area is the formula (25). The higher energy of cliques is similar to higher temperature, but the relation is not direct. The estimation of the temperature and Curie temperature calculation, based on the observations of spins orientation (images), is not simple (Geman and McClure, 1987).

4.6. Layers of an object. Layers, processed by the SIM (Fig. 3) are unordered, but they are significantly correlated, because upper layers are subsets of bottom layers:

$$
\cdots X^{T+1} \subset X^{T} \subset X^{T-1} \cdots .
$$

Unordered processing of layers is the main drawback of the SIM, because the regression line is fitted for both branches together, which is incorrect. The layers are ordered and the threshold is the parameter, so plotting the curve, similar to the magnetization curve is possible (cf. Figs. 11 and 6.

Successive cutting of an object gives layers that are different or identical. Top layers are related to the object with multiple islands. The size and perimeter increase for next layers and the linear function of area-perimeter on a double logarithmic plot could be obtained for some classes of objects,. The connection between the island on lower layers disturbs such relations and nonlinear regions on the area-perimeter plot are observed.

The Glauber dynamics are applied to every pixel. State switching (pixel value change) is related to the specific time moment. The layers subset relation (26) is not guaranteed if the Glauber dynamics simulation is applied. The layers depend on the temperature, but they are very specific realizations of the stochastic evolving process.

4.7. Pseudo-Curie point. We introduce now the pseudo-Curie point $T_{C}^{*}$, because the direct relation between the perimeter and temperature is not available. The pseudo-Curie point is the equilibrium of the pixels of the specific layer and grayscale image, because it is related to the specific threshold $T$.

The proposed pseudo-Curie point is related to the position of the maximum of the function $P=f(A)$ obtained from $A=f(P)$ :

$$
T_{C}^{*}(P, A)=(\max f(P), A(\max f(P)) .
$$

The Curie point is quite often normalized for the Ising model. We use an unnormalized pseudo-Curie point. The normalization of the temperature and magnetization could be a source of information lost, which is not desired. Normalization in both the axes (perimeter and area) depends on the application. The Ising model for the case without an external magnetic field has a unique solution, so Fig. 11 and the Curie temperature could be obtained. More complex structures give different curves. The shape of the magnetization curve could be used for the analysis of unknown material. The differences between shapes ( $M=f(T)$ ) are important for classification purposes. The function $A=f(T)$ could be used instead of $M=$ $f(T)$, due to the above-mentioned problem of temperature estimation for a particular layer.

\section{Application of the area-perimeter relation of the Ising model to the preclassification of cell nuclei}

5.1. Cervical cancer. Cervical cancer is one of the most important cancer types (Cibas and Ducatman, 2009; Chosia and Domagała, 2010). It is an important problem from medical, social and psychological points of view. The cervical cancer screening process is based on a few analyses. Modern reports of cervical cytology are based on the Bethesda classification system of 1998, revisited in 2001 (Solomon and Nayar, 2004).

The most important is the Papanicolaou process (Cibas and Ducatman, 2009; Chosia and Domagała, 2010; Hoda and Hoda, 2007). 
The conventional Papanicolaou process requires professional analysis of very complex microscopic images (Kuehnel, 2003). The lack of process standardization and the different quality of acquired samples are very important problems that additionally influence the variance of biological samples. There are alternatives, like Liquid Based Cytology (LBC) (Sawaya and Sox, 2007; Sykes et al., 2008). It is very well fitted to automatic image analysis, due to the high quality of images and separation of cells by the centrifugal process, but some artifacts interesting from the medical point of view are removed. The conventional Papanicolaou process is one of the most complex biomedical image classes. Such desired operations like cell segmentations using image processing algorithms are extremely difficult.

Cancer is related to cell nuclei mainly, but many properties of the image are analyzed by cytoscreeners. The learning process of the cytoscreener is long (a few years, typically). Computer aided analysis of cell nuclei should improve the detection ratio. Even a single cell is important from a medical point of view and allows the detection of early stages of cancer. Such a preclassification system should be used for rapid analysis of digital images of smear obtained by the transmissive microscope or slide scanner.

The analysis of the cell nuclei texture using fractals is an active research area (Metze, 2013; 2010; Dey and Banik, 2012). Nuclei of melanoma cells are analyzed by Bedin et al. (2010) using a 3D box-counting algorithm. The Minkowski-Bouligand dimension analysis of the B-cells of acute lymphoblastic leukemia is considered (Adam et al., 2006), along with multiple myeloma cells (Ferro et al., 2011). Oral squamous cell carcinoma images are processed using box-counting (Goutzanis et al., 2008). Lacunarity is applied for fractal analysis of early ovarian cancer (Nielsen et al., 2005).

Breast cytology images are considered in many papers, and some algorithms are valuable for cervical cytology analysis. Different techniques are used together, like the shape of the cell, e.g., area, perimeter, convexity, eccentricity and simple texture measure, based on the average value of the red channel (Jelen et al., 2008). The segmentation of cell nuclei is possible using many algorithms: the watershed algorithm, active contours, cellular automata, the grow-cut technique, fuzzy sets of the I and II types, the sonar-like method (Obuchowicz et al., 2008; Hrebień et al., 2008), Hough transform and (1+1) search strategy (Filipczuk et al., 2012; Hrebień et al., 2007), swarm optimization and multi-level thresholding (Kowal et al., 2013).

Much more complex types of images are in cervical cytology and the segmentation process is related to cytoplasm and the cell nucleus. Both of them are analyzed by cytoscreeners. The cytoplasm of a single cell could be deformed or overlapped by other cells, so the overall process is very complex. There is research related to the segmentation of cytoplasm (Walker, 1997; McKenna, 1994). Separated cell nuclei could be processed as binary images (Sedivy et al., 1999).

We use the database of separate cell nuclei classified as correct and atypical (there are many types of atypical cell nuclei). There are 91 of correct and 59 of atypical cells nuclei from a single pathomorphology laboratory in our database. Images were acquired using an AxioCamMRc5 color camera, which supports the $2584 \times 1936$ resolution (about $5 \mathrm{M}$ pixels).

Every image is RGB coded (16-bit/channel, uncompressed). The selection of the color channel is based on the color of the cell nucleus region. The blue channel is extracted if the cell and cytoplasm are blue. The red channel is extracted if the cell and cytoplasm are red. The selected channel is normalized using the following formula:

$$
X=\frac{X_{\text {org }}-\min \left(X_{\text {org }}\right)}{\max \left(X_{\text {org }}\right)-\min \left(X_{\text {org }}\right)},
$$

so values are from the range $X \in[0,1]$.

The color is related to the Papanicolaou process and the age of a particular cell. The cell age and estimated parameter relations are not considered in this paper. All images are obtained at a $400 \times$ magnification (objective $40 \times$ ), which is quite high for the optical microscope. The $100 \times$ magnification is used in the screening process typically. Higher magnification $(400 \times)$ is used for the cells that need a more detailed investigation.

Some researchers have good or poor results of atypical cell nuclei preclassification. It is very hard to compare the results, because most of the works are based on a low resolution camera (VGA typically, color or monochrome sensors) and lower magnifications (e.g., $100 \times)$.

In our previous work (Oszutowska-Mazurek and Mazurek, 2012) we described how low resolution and low magnification together influence detection results even for the simplest cases.

There are many types of atypical cells due to the boundary shape, but limited availability of smears with atypical cells is the reason for the binary preclassification assumption. Hypothetically, such binary classification is sufficient for image analysis. Another hypothesis is related to the number and type of parameters that should be used.

Cancerous changes are related to cell nuclei, so they could be observed directly as differences related to the cell size, boundary or texture. Secondary effects typical for cancer, like specific clusters of cells, are visible in Papanicolaou smears.

The most interesting is the texture of cell nuclei, because it is related to the large part of the cell 


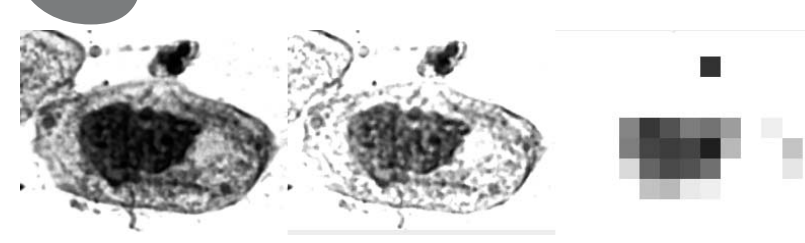

Fig. 12. Example of a cell nucleus (left), blue channel (middle), low resolution of the blue channel (right).

nucleus. The boundary of the cell nucleus is analyzed by cytoscreeners, but is difficult to analyse if the image quality is low. Precised segmentation of cell nuclei is not possible (Fig. 12) sometimes (bottom-left area of cell nuclei, in particular). The texture is more independent on such artifacts, so a cell nucleus area as large as possible should be used.

5.2. Area-perimeter analysis. Area-perimeter covers more information about texture than a single FD value. Noise related to acquisition influences results. There is also noise related to the texture. A specific texture gives different area-perimeter trajectories. The area of the object is the area for the first or last layer:

$$
A=-A_{\min }=A_{\max } .
$$

The pseudo-Curie point is a possible descriptor of texture, but it is insufficient for our database. We propose the polynomial approximation of the area-perimeter curve for Papanicolaou cell nucleus image analysis. This is an approximation of empirical measurements. Multiple observations with the same area and perimeter values are rejected, so all area and perimeter pairs are unique for the particular curve. A few variants of the polynomial were tested (different orders and coefficient sets). The best results are obtained for the third order of the polynomial.

Four coefficients $\left\{k_{0}, k_{1}, k_{2}, k_{3}\right\}$ are fitted, using the minimal Mean Square Error (MSE) criteria for every curve (every nucleus):

$$
\hat{P}(a)=k_{3} a^{3}+k_{2} a^{2}+k_{1} a+k_{0},
$$

where $a$ is the specific area and $k_{0}$ is related to the pseudo-Curie point position $T_{C}^{*}(P, A)=$ $(\max f(P), A(\max f(P))$ :

$$
k_{0} \sim \max f(P) .
$$

Three parameters are used for the cell nucleus $\left\{k_{0}, k_{1}, k_{2}\right\}$ description. The $k_{3}$ coefficient is rejected because separation for $\left\{k_{0}, k_{1}, k_{3}\right\},\left\{k_{0}, k_{2}, k_{3}\right\}$ and $\left\{k_{1}, k_{2}, k_{3}\right\}$ sets is less significant, which was empirically tested.

The reduction of the number of parameters to two, does not give good separation. The second order polynomial, where are only three parameters available $\left\{k_{0}, k_{1}, k_{2}\right\}$, does not enable sufficient separation, which was empirically tested. In Fig. 13 we show an example result for the approximation of the area-perimeter curve.

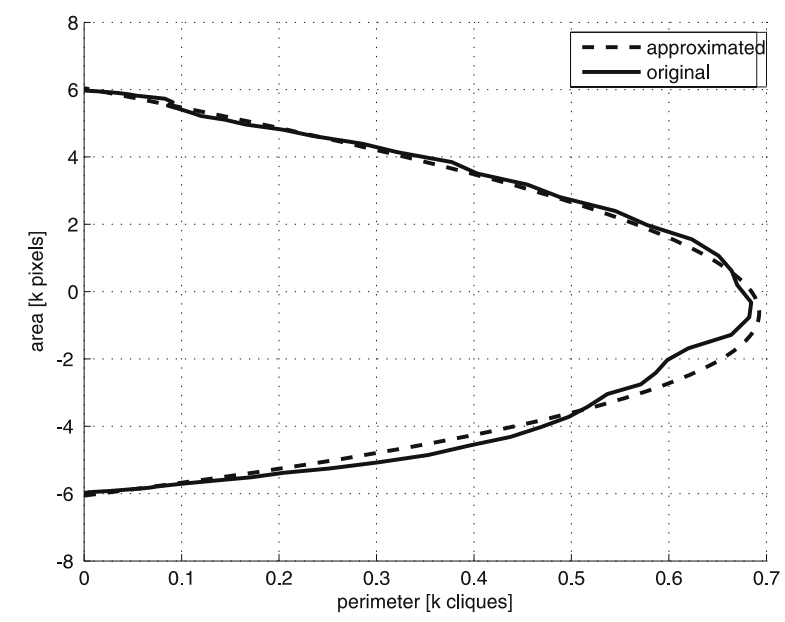

Fig. 13. Area-perimeter relation and the approximation curve.

Area-perimeter relations for atypical cell nuclei are shown in Fig. 14 The pseudo-Curie point is not located on $A=0$ line, which is clearly visible in the largest curve.

The reason for the visible gap between correct and atypical cells is the fact that correct cell nuclei are usually smaller (Fig. 15). The most important for the selection of the preclassification algorithm are curves related to atypical cell nuclei, which are located in the correct cells nuclei area (Fig. 15).

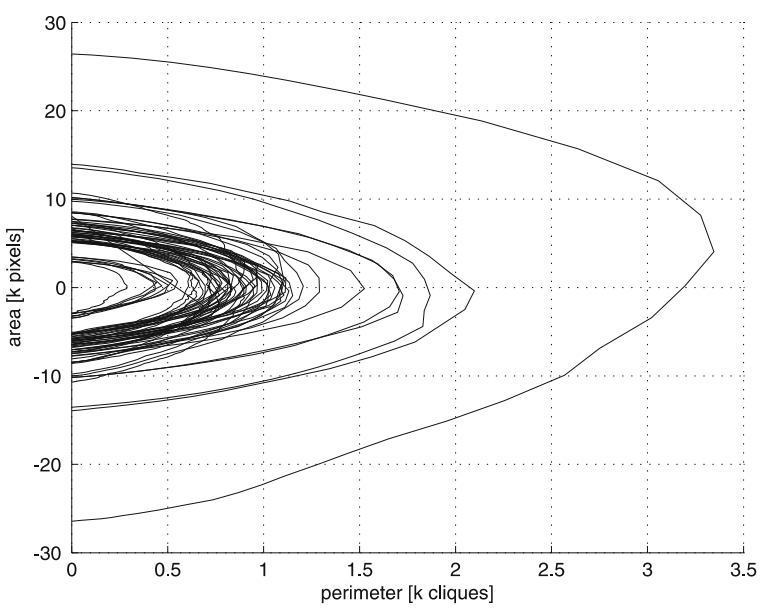

Fig. 14. Area-perimeter relation for atypical cell nuclei.

The polynomial approximation allows the filtering of the curves. The results of filtration are shown in Figs. 16 and 17 .

The obtained parameters of the curve $\left\{k_{0}, k_{1}, k_{2}\right\}$ could be used for the preclassification of the cells using the texture of the cell nuclei only. The separation of the 


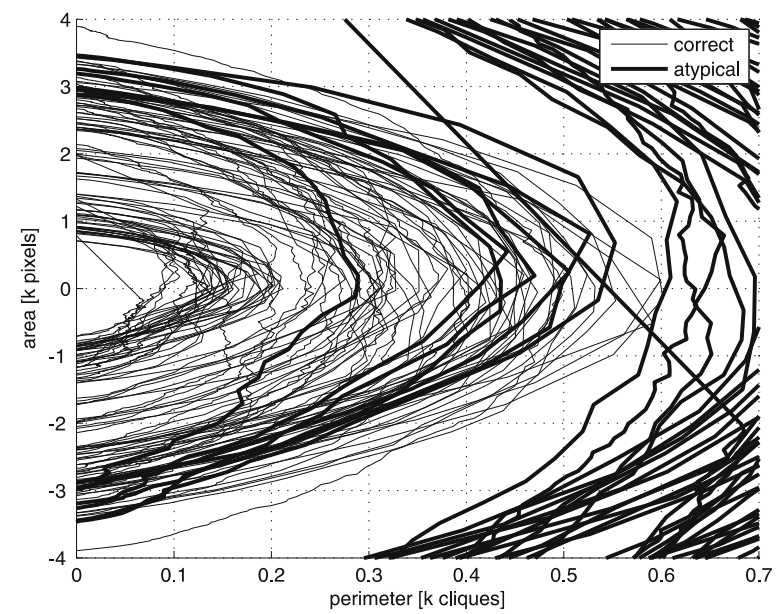

Fig. 15. Area-perimeter relation for correct cell nuclei. Atypical area-perimeter relations for atypical cells nuclei are shown partially.

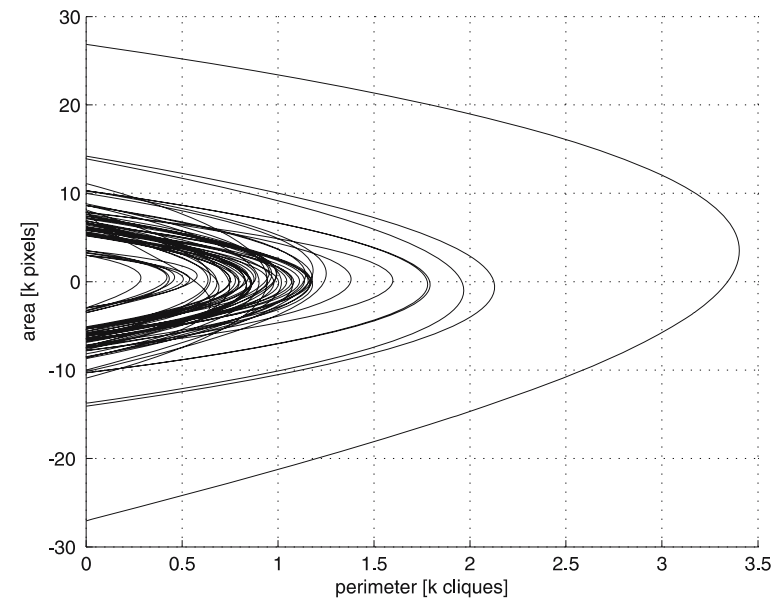

Fig. 16. Area-perimeter relation for atypical cell nuclei (polynomial approximation).

correct and atypical cells nuclei for the database entries is shown in Fig. 18, It is clearly visible that three parameters are sufficient for this database.

5.3. Empirical sensitivity. The effects of cell nuclei area reduction due to the segmentation problem of blurred edges could be analyzed using mask reduction. Morphological erosion using a circular Structuring Element (SE) is applied for every mask of cell nuclei. Reduction using $\mathrm{SE}=2$ and $\mathrm{SE}=5$ is shown in Figs. 19 and 20, respectively. The case $\mathrm{SE}=5$ of is related to a significant reduction in the area of cell nuclei. The largest correct cell nuclei has an area of 4323 pixels at $\mathrm{SE}=0$ and is reduced to 3346 pixels at $\mathrm{SE}=5$, which is a significant $(30 \%)$ reduction. Even such a large reduction of cell areas allows classification.

There are two main populations of atypical cell

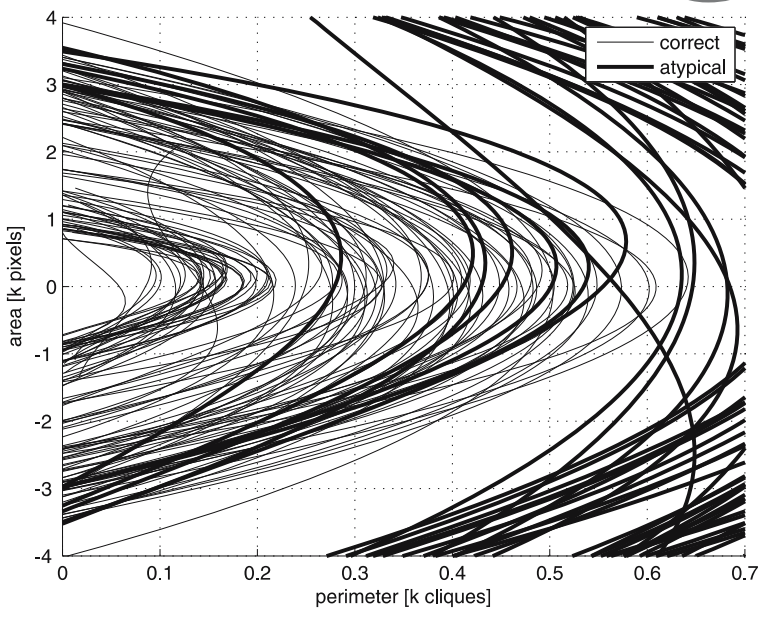

Fig. 17. Area-perimeter relation for correct cell nuclei (polynomial approximation). Atypical area-perimeter relations for atypical cell nuclei are shown partially.

nuclei. The first one could be characterized by a high value of the ratio $-k_{2} / k_{0}$, which corresponds to large sizes of the cell nuclei area $\left(k_{0}\right)$. The most important is the separation of the second population, which has ratio of the values $-k_{2} / k_{0}$ similar to the same correct cells nuclei. This very important population could be separated using the relation $k_{1} / k_{2}$.

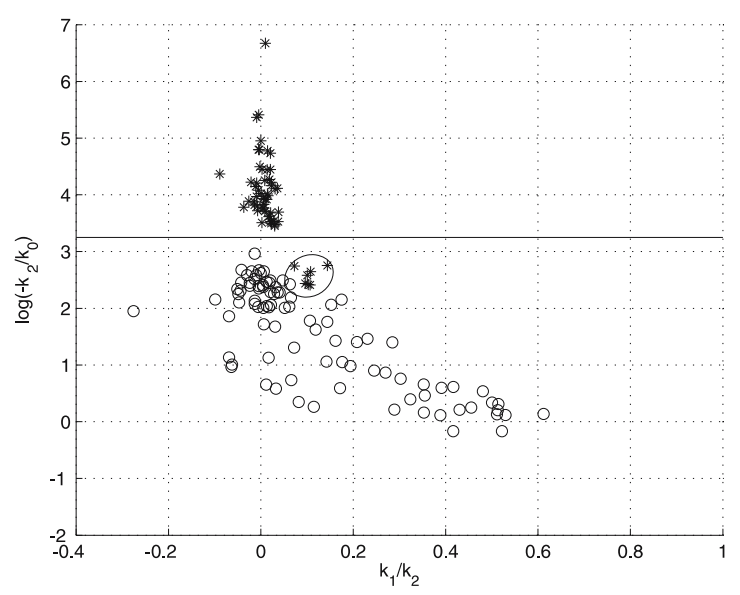

Fig. 18. Separation of correct and atypical cell nuclei (starsatypical, circles-correct cell nuclei): the best mask estimated manually.

The cell nuclei segmentation algorithm is beyond the scope of this paper. The impact of the sensitivity of the proposed analysis technique on classification could be analyzed empirically.

First of all, we assume the availability of a high quality segmentation algorithm, which is comparable 


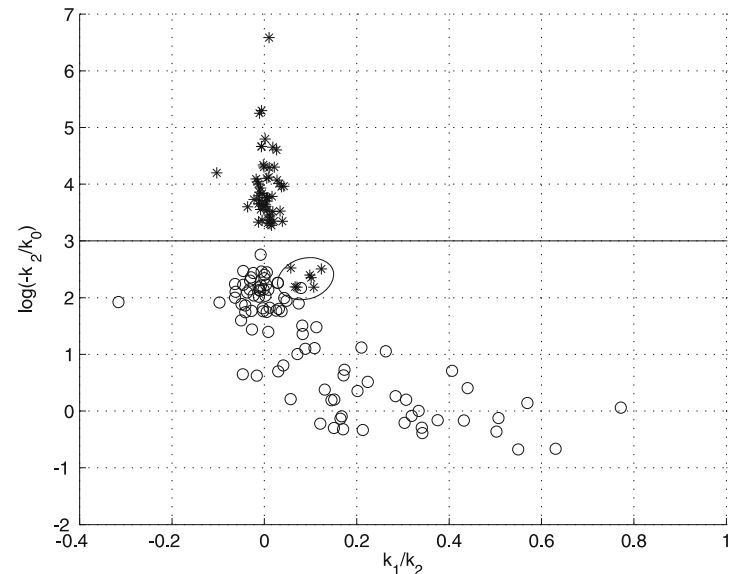

Fig. 19. Separation of correct and atypical cell nuclei (starsatypical, circles—correct cell nuclei): the best mask estimated manually eroded by 2 pixels.

with human based segmentation. Such segmentation is sufficient and the separation results are depicted in Fig.18. Apart from that, a simple segmentation algorithm could be based on local spatial analysis of the mean optical density (Fig. 12). The area of cell nucleus is dark and the cytoplasm is highly transmissive and bright. The segmentation algorithm based on this knowledge could estimate the area of the cell nuclei center, even if the image is blurred due to biological artifacts. The boundary area of cell nuclei, which is hard to assign to the cell nuclei or the cytoplasm region, could be omitted in the analysis. In such a case only the inner part of the cell nucleus is processed by the image analysis algorithm and some estimator values are obtained.

The reduction of the cell nuclei area influences the separation depending on the significance of this area and the selection of estimators, especially for a small size object. A relative reduction in the small size cell nuclei is significant in such cases (Figs. 19 and 20).

Simple classification, based on clustering related to atypical cells, could be proposed. The area of the largest population of atypical cells could be defined using a linear discriminant function. This line could be located between the smallest atypical cell of this population and largest the cell of correct population using the ratio $-\log \left(k_{2} / k_{0}\right)$. The second area of the atypical cells population could be defined using an ellipsoid discriminant of a $95 \%$ confidence region. The distribution cannot be determined correctly for the second area due to very small sample size (7 cases). Both discriminants are shown in Figs. $18,+20$, The number of correct cells, which are inside defined areas of atypical cells, is very small and amounts to 1,2 and 7, respectively. Such cell nuclei and correctly

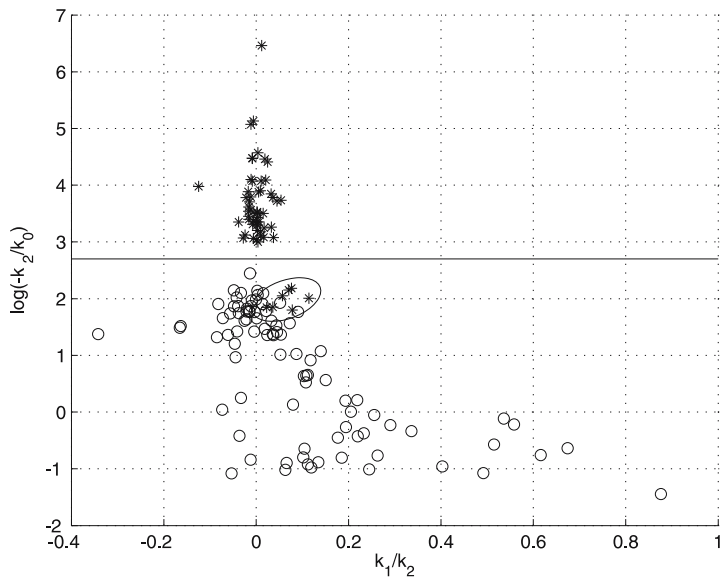

Fig. 20. Separation of correct and atypical cell nuclei (starsatypical, circles-correct cell nuclei): the best mask estimated manually eroded by 5 pixels.

detected atypical cell nuclei should be considered by the cytoscreener immediately.

The reduction in the cell nuclei area is unknown for the algorithm, and cumulative separation could be computed. The results for the original mask and two reduced cases (erosion by 2 and 5 pixels) are shown in Fig. 21,

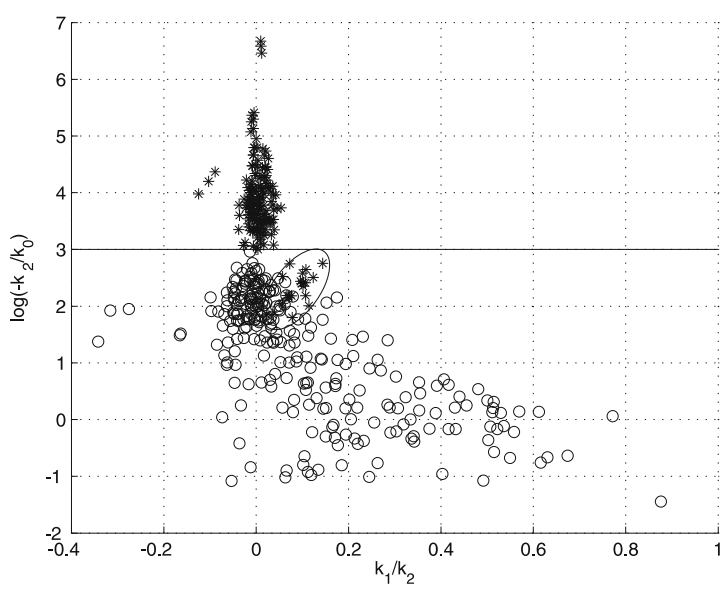

Fig. 21. Separation of correct and atypical cell nuclei (starsatypical, circles - correct cell nuclei): the best mask estimated manually eroded by 0,2 and 5 pixels.

The number of atypical cases in the elliptical area is 21 . The number of correct cases misclassified as a typical is only 23 (it is $8 \%$ of the correct cell nuclei population). 


\section{Discussion and conclusions}

The application of FD analysis using the SIM is limited to the part of the area-perimeter curve for a small perimeter only if the layers are created by successive cutting. This is the main limitation of the SIM, which cannot be applied correctly for such objects. Incorrect results could be obtained, which was shown in illustrative examples (a negative or infinite fractal dimension).

The reformulation of the SIM in the context of the Ising model is possible. The magnetization curve allows the description of such materials. Instead of magnetization, which is temperature dependent, we proposed direct application of area-perimeter analysis, with a pseudo-Curie point. The generalization of this point (a single parameter is not enough for the description of some objects) is the approximation curve using a polynomial. A few coefficients (weights) of the polynomial are sufficient for the description of the curve. These coefficients are related, but not directly, to the variability of the FD for different layers. The proposed technique could be applied for other types of objects.

The obtained results are not a clinical proof of the correctness of the proposed technique for the analysis of cell nuclei of conventional Papanicolaou smears. These results show that it is possible to use such a technique separately or together with other techniques for the detection of atypical cells. A reliable verification needs a very large database of images obtained from many laboratories, which is not available for the authors. The comparison of other methods is discussed widely by Oszutowska-Mazurek (2013).

It should be noted that separated cells with quite a high quality image are intentionally selected for research purposes. The results show the ability of the application of the proposed technique. The area of the analysis for the proposed technique is irregular, which is very important for biological objects. Typical fractal estimator algorithms, dedicated to textures, require a square area, excluding the variogram algorithm. Computations of the perimeter and area for multiple layers are possible using parallel processing systems (SIMD processors, DSPs, FPGAs, GPGPUs). Parallel computations are possible because layers are separated. This is a very fast approach for computing devices available nowadays. This algorithm is based on very simple local morphological filers (cliques detection), so memory consumption is very low. The variogram algorithm requires comparison of the height (values) between every pair of pixels. The variogram requires a lot of memory access that is non-local, which adds unnecessary delays.

It was preliminarily tested that the reduction of the area of analysis does not influence significantly the reduction of separation between the correct and atypical cells. The analysis of sensitivity is shown in the paper.
Another interesting research area is the analysis of non-separated cells. Cervical cancer cells very often are grouped in clusters. Testing the ability of preclassification of such clusters is important from the practical point of view.

\section{Acknowledgment}

This work is supported by the UE EFRR ZPORR project Z/2.32/I/1.3.1/267/05 Szczecin University of Technology_Research and Education Center of Modern Multimedia Technologies (Poland).

The authors wish to thank Kinga Sycz, M.D., Ph.D. (Director of the Pathomorphology Department in the Independent Public Voivodeship United Hospital) for access to Papanicolaou slides of cervical cytology.

\section{References}

Adam, R., Silva, R., Pereira, F., Leite, N., Lorand-Metze, I. and Metze, K. (2006). The fractal dimension of nuclear chromatin as a prognostic factor in acute precursor B lymphoblastic leukemia, Cellular Oncology 28(1-2): 55-59.

Atkinson, P. (2002). Spatial statistics, in A. Stein, F. Van Der Meer and B. Gorte (Eds.), Spatial Statistics for Remote Sensing, Kluwer Academic Publishers, Boston, MA, pp. 57-82.

Barnsley, M., Devaney, R., Mandelbrot, B., Peitgen, H.-O., Saupe, S. and Voss, R. (1988). The Science of Fractal Images, Springer-Verlag, Heidelberg.

Bedin, V., Adam, R., de Sa, B., Landman, G. and Metze, K. (2010). Fractal dimension of chromatin is an independent prognostic factor for survival in melanoma, $B M C$ Cancer 10(260), 6 pages.

Binney, J., Dowrick, N., Fisher, A. and Newman, M. (1992). The Theory of Critical Phenomena. An Introduction to the Renormalization Group, Clarendon Press, Oxford.

Chosia, M. and Domagała, W. (2010). CCervical Cytodiagnosis, Pro Pharmacia Futura, Warsaw, (in Polish).

Cibas, E. and Ducatman, B. (2009). Cytology. Diagnostic Principles and Clinical Correlates, Saunders Elsevier, Philadelphia, PA.

Clarke, K. (1986). Computation of the fractal dimension of topographic surfaces using the triangular prism surface area method, Computer and Geoscience 12(5): 713-722.

Costa, L. and Cesar Jr., R. (2009). Shape Classification and Analysis. Theory and Practice, CRC Press, Boca Raton, FL.

Dey, P. and Banik, T. (2012). Fractal dimension of chromatin texture of squamous intraepithelial lesions of cervix, Diagnostic Cytopathology 40(2): 152-154.

Engler, O. and Randle, V. (2010). Introduction to Texture Analysis: Macrotexture, Microtexture, and Orientation Mapping, CRC Press, Amsterdam. 
Ferro, D., Falconi, M., Adam, R., Ortega, M., Lima, C., de Souza, C., Lorand-Metze, I. and Metze, K. (2011). Fractal characteristics of May-Grünwald-Giemsa stained chromatin are independent prognostic factors for survival in multiple myeloma, PLOS ONE 6(6): 1-8.

Filipczuk, P., Wojtak, W. and Obuchowicz, A. (2012). Automatic nuclei detection on cytological images using the firefly optimization algorithm, in E. Piętka and J. Kawa (Eds.), Information Technologies in Biomedicine, Lecture Notes in Computer Science, Vol. 7339, Springer, Heidelberg, pp. 85-92.

Geman, S. and McClure, D. (1987). Statistical methods for tomographic image reconstruction, Bulletin of the International Statistical Institute LII(4): 5-21.

Glauber, R. (1963). Time-dependent statistics of the Ising model, Journal of Mathematical Physics A 4(2): 1299-1303.

Goodchild, M. (1980). Fractals and the accuracy of geographical measures, Mathematical Geology 12(2): 85-98.

Goutzanis, L., Papadogeorgakis, N., Pavlopoulos, P., Katti, K., Petsinis, V., Plochoras, I., Pantelidaki, C., Kavantzas, N., Patsouris, E. and Alexandridis, C. (2008). Nuclear fractal dimension as a prognostic factor in oral squamous cell carcinoma, Oral Oncology 44(4): 345-353.

Harte, D. (2001). Multifractals. Theory and Applications, Chapman \& Hall/CRC, Boca Raton, FL.

Hoda, R. and Hoda, S. (2007). Fundamentals of Pap Test Cytology, Humana Press, Totowa.

Hrebień, M., Korbicz, J. and Obuchowicz, A. (2007). Hough transform, $(1+1)$ search strategy and watershed algorithm in segmentation of cytological images, in M. Kurzyński, E. Puchała, M. Woźniak and A. Żołnierek (Eds.), Computer Recognition Systems, Advances in Soft Computing, Vol. 45, Springer, Berlin, pp. 550-557.

Hrebień, M., Steć, P., Nieczkowski, T. and Obuchowicz, A. (2008). Segmentation of breast cancer fine needle biopsy cytological images, International Journal of Applied Mathematics and Computer Science 18(2): 159-170, DOI: 10.2478/v10006-008-0015-x.

Huang, Z., Tian, J. and Wang, Z. (1990). A study of the slit island analysis as a method for measuring fractal dimension of fractured surface, Scripta Metall Mater 24(6): 967-972.

Jeleń, L., Fevens, T. and Krzyżak, A. (2008). Classification of breast cancer malignancy using cytological images of fine needle aspiration biopsies, International Journal of Applied Mathematics and Computer Science 18(1): 75-83, DOI: 10.2478/v10006-008-0007-x.

Kaye, B. (1994). A Random Walk through Fractal Dimensions, $\mathrm{VCH}$, Weinham/New York, NY.

Kowal, M., Filipczuk, P., Marciniak, A. and Obuchowicz, A. (2013). Swarm optimization and multi-level thresholding of cytological images for breast cancer diagnosis, in R. Burduk, K. Jackowski, M. Kurzyński, M. Woźniak and A. Żołnierek (Eds.), CORES 2013, Advances in Intelligent Systems and Computing, Vol. 226, Springer-Verlag, Heidelberg, pp. 611-620.
Kuehnel, W. (2003). Color Atlas of Cytology, Histology, and Microscopic Anatomy, Thieme, New York, NY.

Loncaric, A. (1998). A survey of shape analysis techniques, Pattern Recognition 31(8): 983-1001.

Lu, C. (1995). On the validity of the slit islands analysis in the measure of fractal dimension of fracture surfaces, International Journal of Fracture 69(4): 77-80.

Mandelbrot, B. (1983). The Fractal Geometry of the Nature, W.H. Freeman and Company, New York, NY.

Mandelbrot, B., Passoja, D. and Paullay, A. (1984). Fractal character of fracture surfaces of metals, Nature 308: 721-722.

McKenna, S. (1994). Automated Analysis of Papanicolaou Smears, Ph.D. thesis, University of Dundee, Dundee.

Metze, K. (2010). Fractal dimension of chromatin and cancer prognosis, Epigenomics 2(5): 601-604.

Metze, K. (2013). Fractal dimension of chromatin: Potential molecular diagnostic applications for cancer prognosis, Expert Review of Molecular Diagnostics 13(7): 719-735.

Mingqiang, Y., Kidiyo, K. and Ronsin, J. (2008). A survey of shape feature extraction techniques, in P.-Y. Yin (Ed.), Pattern Recognition Techniques, Technology and Applications, InTech, Rijeka, pp. 43-90.

Nielsen, B., Albregtsen, F. and Danielsen, H. (2005). Fractal analysis of monolayer cell nuclei from two different prognostic classes of early ovarian cancer, in G.A. Losa, D. Merlini, T.F. Nonnenmacher and E.R. Weibel (Eds.), Fractals in Biology and Medicine, Vol. 4, Birkhäuser, Boston, MA, pp. 175-186.

Obuchowicz, A., Hrebień, M., Nieczkowski, T. and Marciniak, A. (2008). Computational intelligence techniques in image segmentation for cytopathology, in T.G. Smoliński, M.G. Milanova and A.-E. Hassanien (Eds.), Computational Intelligence in Biomedicine and Bioinformatics, Studies in Computational Intelligence, Vol. 151, Springer, Berlin, pp. 169-199.

Oszutowska, D. and Purczyński, J. (2012). Estimation of the fractal dimension using tiled triangular prism method for biological non-rectangular objects, Electrical Review R.88 (10b): 261-263.

Oszutowska-Mazurek, D. (2013). Parameter Estimation of Microscopic Objects Using Algorithms of Digital Image Processing for the Purpose of Cytomorphometry, Ph.D. thesis, West-Pomeranian University of Technology, Szczecin, (in Polish).

Oszutowska-Mazurek, D. and Mazurek, P. (2012). Analysis of influence of cell nuclei segmentation in Papanicolaou smears on fractal dimension measurements, Measurement Automation and Monitoring 58(6): 498-501, (in Polish).

Oszutowska-Mazurek, D., Mazurek, P., Sycz, K. and Wójciuk, G.-W. (2012). Estimation of fractal dimension according to optical density of cell nuclei in Papanicolaou smears, in E. Piętka and J. Kawa (Eds.), Information Technologies in Biomedicine, Lecture Notes in Computer Science, Vol. 7339, Springer, Heidelberg, pp. 456-463. 
Oszutowska-Mazurek, D., Mazurek, P., Sycz, K. and Wójciuk, G.-W. (2013). Variogram based estimator of fractal dimension for the analysis of cell nuclei from the Papanicolaou smears, in R.S. Choraś (Ed.), Image Processing and Communications Challenges 4, Advances in Intelligent Systems and Computing, Springer-Verlag, Heidelberg, pp. 47-54.

Parker, J. (1997). Algorithms for Image Processing and Computer Vision, Wiley, Indianapolis, IN.

Peitgen, H., Jürgens, H. and Saupe, D. (1991). Fractals for the Classrooms, Vol. 1, Springer-Verlag, Heidelberg.

Peitgen, H., Jürgens, H. and Saupe, D. (1992). Fractals for the Classrooms, Vol. 2, Springer-Verlag, Heidelberg.

Sawaya, G. and Sox, H. (2007). Trials that matter: Liquid-based cervical cytology: Disadvantages seem to outweigh advantages, Annals Internal Medicine 147(9): 668-669.

Sedivy, R., Windischberger, C., Svozil, K., Moser, E. and Breitenecker, G. (1999). Fractal analysis: An objective method for identifying atypical nuclei in dysplastic lesions of the cervix uteri, Gynecologic Oncology 75: 78-83.

Seuront, L. (2010). Fractals and Multifractals in Ecology and Aquatic Science, CRC Press, Boca Raton, FL.

Skomski, R. (2008). Simple Models of Magnetism, Oxford University Press, Oxford.

Smereka, M. and Dulęba, I. (2008). Circular object detection using a modified Hough transform, International Journal of Applied Mathematics and Computer Science 18(1): 85-91, DOI: 10.2478/v10006-008-0008-9.

Śmietański, J., Tadeusiewicz, R. and Łuczyńska, E. (2010). Texture analysis in perfusion images of prostate cancer-A case study, International Journal of Applied Mathematics and Computer Science 20(1): 149-156, DOI: 10.2478/v10006-010-0011-9.

Solomon, D. and Nayar, R. (2004). The Bethesda System for Reporting Cervical Cytology, Springer, New York, NY.

Steven, I. (1993). Linear Richardson plots from non-fractal data sets, Dutch Mathematical Geology 25(6): 737-751.

Styer, D. (2007). Statistical Mechanics, Oberlin College, Oberlin.

Sun, W. (2006). Three new implementations of the triangular prism method for computing the fractal dimension of remote sensing images, Photogrammetric Engineering \& Remote Sensing 72(4): 372-382.

Sykes, P., Harker, D., Miller, A., Whitehead, M., Neal, H., Wells, J. and Peddied, D. (2008). A randomised comparison of SurePath liquid-based cytology and conventional smear cytology in a colposcopy clinic setting, General Gynaecology 115(11): 1375-1381.
Voss, R. (1988). Fractals in nature: From characterization to simulation, in H.-O. Peitgen and D. Saupe (Eds.), The Science of Fractal Images, Springer-Verlag, Heidelberg, pp. 21-70.

Walker, R. (1997). Adaptive Multi-Scale Texture Analysis with Applications to Automated Cytology, Ph.D. thesis, University of Queensland, Brisbane.

Wen, R. and Sinding-Larsen, R. (1997). Uncertainty in fractal dimension estimated from power spectra and variogram, Mathematical Geology 29(6): 727-753.

Zhou, G. and Lam, N.-N. (2005). A comparison of fractal dimension estimators based on multiple surface generation algorithms, Computers \& Geosciences 31(10): 1260-1269.

Zieliński, K. and Strzelecki, M. (2002). Computer Biomedical Image Analysis. Introduction to Morphometry and Quantitative Pathology, PWN, Warsaw, (in Polish).

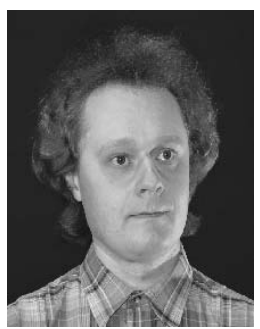

Przemysław Mazurek received the M.Sc. and Ph.D. degrees in electrical engineering from the Szczecin University of Technology, Poland, in 1998 and 2002, respectively. He is currently with the Department of Signal Processing and Multimedia Engineering, West-Pomeranian University of Technology, Szczecin. He is an author of more than 130 technical papers. His current research activities include motion tracking, track-beforedetect systems, HCI systems and image processing in medical applications.

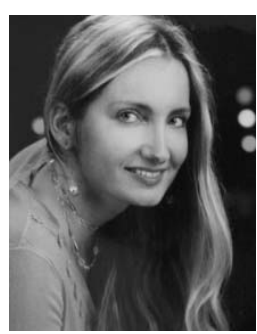

Dorota Oszutowska-Mazurek received her M.Sc. in biology from Szczecin University, Poland, in 2004. She is currently with the Department of Pathomorphology, Gryfice Medicam Hospital, Poland. Her current research activities include image processing in medical applications.

Received: 5 February 2013 Revised: 13 August 2013 